\title{
Activation Kinetics of Retinal Cones and Rods: Response to Intense Flashes of Light
}

\author{
Shaul Hestrin and Juan I. Korenbrot \\ Department of Physiology, School of Medicine, University of California at San Francisco, San Francisco, California 94143
}

\begin{abstract}
Cone photoreceptors are less sensitive to light and the duration of their photoresponse is shorter than that of rods. In salamander rods and cones, we identified 3 components in membrane currents activated by bright flashes of light: an early receptor current (ERC) resulting from charge displacement within visual pigments, a saturation photocurrent generated by the closure of the cGMP-sensitive channels, and a putative $\mathrm{Na}-\mathrm{Ca}$ exchanger current. The time courses of both the ERC and the onset of the saturation photocurrent were similar in rods and cones. The putative $\mathrm{Na}-\mathrm{Ca}$ exchanger current, on the other hand, is 4- to 8-fold faster in cones. The onset of the saturation photocurrent consisted of a delay followed by a fast relaxation with an exponential time course. In both photoreceptor types the delay and the time course of the fast relaxation are dependent on light intensity and reach a limiting value when about $1 \%$ of the photopigment is bleached. The limiting value of the delay, about $8 \mathrm{msec}$, and of the relaxation time constant, about 2 msec, are nearly identical in rods and cones. The near identity of these parameters implies that at least 2 kinetic steps in the activation response of rods and cones are quantitatively similar. These findings suggest that the functional differences between rods and cones may arise from disparities in the processes that restore the components of the phototransduction cascade to their dark level and not from differences in the activation processes.
\end{abstract}

The visual system of man can detect single photons in darkness and yet remain functional under bright lights (Rodieck, 1973). This flexible performance arises from the presence in the vertebrate retina of rod and cone photoreceptors which have overlapping, but not identical operating ranges. Cones are less sensitive to light than rods, and, furthermore, their response adapts over a much wider range of light; consequently, cones can operate under bright light that saturates the rod response (Baylor, 1987).

While the processes that generate the functional difference between rods and cones have not been identified, it appears that the mechanism of phototransduction is not radically different in the 2 types of receptors. Light detection in both rods and cones is initiated by excitation of structurally homologous photopigment molecules located in the outer segments (Applebury

\footnotetext{
Received Aug. 10, 1989; revised Dec. 26, 1989; accepted Jan. 23, 1990.

We would like to thank Drs. Joe Howard, Timothy Kraft, and Jim Miller for reading the manuscript. This work was supported by NIH grants EY07507 to S.H. and EY05617 to J.I.K.

Correspondence should be addressed to Shaul Hestrin at the above address.

Copyright (c) 1990 Society for Neuroscience $0270-6474 / 90 / 061967-07 \$ 02.00 / 0$
}

and Hargrave, 1986; Nathans, 1987). The excited photopigment molecules activate GTP-binding proteins (Strycr, 1986; Pugh, 1987). A high level of homology, but not identity, has been found in the sequences of cDNA coding the GTP-binding proteins from rods and cones (Lerea et al., 1986; Grunwald et al., 1986). The activated GTP-binding proteins, in turn, activate cGMP-specific phosphodiesterases (PDEs). Light-sensitive PDEs have been isolated from both rods and cones, and their biochemical characteristics are generally similar (Hurwitz et al., 1985; Booth et al., 1986; Gillespie and Beavo, 1988; Orlov et al., 1988). The cGMP-sensitive channels of rods and cones are generally similar in their electrophysiological properties (Fesenko et al., 1985; Haynes and Yau, 1985).

If the major proteins in the cGMP cascade in rods and cones are similar, what then is the mechanism that generates their different functional behavior? The differences between rods and cones could be the result of differences in the detailed properties of the individual proteins or in differences in their control, in their number in the outer segment, or in geometrical and morphological considerations.

Several lines of evidence suggest that the photocurrent time course reflects light-dependent changes in cytoplasmic cGMP (Stryer, 1986; Pugh, 1987). The time course of the photocurrent in response to dim light stimulation depends on the activity of both PDE and guanylate cyclase. Thus, the differences of the dim flash response of rods and cones could be related to the activity of the PDE or the guanylate cyclase or both. Under intense lights, the maximum hydrolytic velocity of the PDE can reach levels of several hundreds of millimolars CGMP per second, whereas the maximum velocity of cGMP synthesis is only several tens of micromolars per second (see Pugh, 1987). Thus, the initial time course of the response to intense light stimulation should be independent of cGMP synthesis, reflecting only the kinetics of cGMP hydrolysis and the interaction of cGMP with the cGMP-sensitive channels. Comparison of the response to bright lights of rods and cones, therefore, can provide insight into the molecular mechanisms of their functional differences. Indeed, Cobbs and Pugh (1987) have previously shown that the kinetics of the response to bright flashes in rods can be explained by the activation of the PDE, the limiting rate of cGMP hydrolysis, and the rate of closure of the CGMP-sensitive channels.

The rate-limiting kinetics of the photocurrent can be measured only under voltage clamp; otherwise, capacitative currents would limit the speed of the measured responses (Cobbs and Pugh, 1987). Cobbs and Pugh (1987) have shown that for rods the kinetics of the photoresponse reaches a limiting value when stimulated with intense light. In cones, the limiting kinetics of the photocurrent generated by intense flashes of light are unknown. Here we report on the response to bright lights in sal- 
amander cones under voltage clamp. We compare these responses to those of rods of the same species in an effort to analyze the mechanisms of the rod-cone difference.

\section{Materials and Methods}

Materials. Larval stage tiger salamanders (Ambystoma tigrinum) were obtained from Lowrance Waterdog Farm and maintained at $4^{\circ} \mathrm{C}$ under $12 \mathrm{hr}$ dark-light cycle. All chemicals were purchased from Sigma Chemical Co. (St. Louis, MO). Minimum essential medium (MEM) amino acids and vitamins were obtained from the cell culture facility at the University of California at San Francisco.

Cells. Single cones and rods were prepared by mechanical dissociation of isolated retinas and were attached to glass coverslips as previously described (Hestrin and Korenbrot, 1987). We recorded from both intact cones and rods and from incomplete cones that included the elipsoid but were missing the nuclear region of the soma.

Electrical recording. The experimental chamber containing the cells was positioned on a microscope stage and observed under infrared illumination $(830 \pm 10 \mathrm{~nm})$ with the aid of a TV camera and monitor. After contact was made between the recording pipette and the cell, the infrared source was turned off to prevent light adaptation. Tight-seal whole-cell recordings were made using aluminosilicate glass pipettes (Corning-1723) coated with Sylgard (Resin 184 Dow Corning, Midland, MI). The pipettes were filled with a solution of the following composition (in mM): $120 \mathrm{HMOPS}, 75 \mathrm{KOH}, 4 \mathrm{MgCl}_{2}, 5 \mathrm{Na}_{2}$ ATP, $2 \mathrm{Na}_{4}$ GTP, 0.05 EGTA. The patch pipettes were filled with solution containing only trace amounts of $\mathrm{Ca}$ buffer because $\mathrm{Ca}$ buffers affect the light response of rods (Korenbrot and Miller, 1986; Torre et al., 1986) and cones (S. Hestrin and J. 1. Korenbrot, unpublished observations). The composition of the cell-bathing solution was (in $\mathrm{mM}$ ): $95 \mathrm{NaCl}, 2 \mathrm{KCl}, 1 \mathrm{CaCl}_{2}$, $2 \mathrm{MgCl}_{2}, 5 \mathrm{NaHCO}_{3}, 10$ glucose, 10 HEPES, MEM amino acids, MEM vitamins, $0.1 \mathrm{mg} / \mathrm{ml} \mathrm{BSA}$. The osmolallity and the $\mathrm{pH}$ of these solutions were 224 , and 7.4 , respectively.

Membrane currents were recorded with a patch-clamp amplifier (EPC7, List-Medical, Darmstadt, FRG). After establishing a tight seal on the inner segment, the capacitance of the pipette and headstage were cancelled. Whole-cell recording mode was initiated by the application of suction. The cell's capacitance was monitored by applying $10 \mathrm{mV}$ command pulses to the pipette. Typically, the capacity current was well fit by a single-exponential function. In some cases, an additional fast component was required to obtain a fit of the charging current. The series resistance was $5-15 \mathrm{M} \Omega$, and the dominant time constant of the capacitative charging was $0.5-2.5 \mathrm{msec}$. In some experiments, series resistance compensation (50-70\%) was applied. In all the experiments, unless otherwise specified, the membrane potential was held at $-40 \mathrm{mV}$ (corrected for the junction potential).

A microcomputer (PDP 11/23, Digital Equipment Corp., Maynard, MA) was used to acquire data and apply command voltages as well as to control light stimuli. Analog data were filtered with an 8-pole Bessel filter (model 902LPF, Frequency Devices, Haverhill, MA). A nonlinear least-square routine was used to curve-fit model functions to experimental data.

Light stimuli. Cells were illuminated with brief flashes of light (2.4$3.0 \mathrm{msec}$ ) generated by a halogen-tungsten source. Flash duration was controlled with a mechanical shutter (model 26L, A.W. Vincent Assoc., Rochester, NY). The stimulating light for the cones was filtered with a narrow-band interference filter $620 \pm 10 \mathrm{~nm}$ (or $500 \mathrm{~nm}$ for the rods) (Ealing Corp., South Natwick, MA). In some experiments, unfiltered "white light" was used. Light intensity was controlled with neutral density filters. Flash intensity was measured with a calibrated photodiode placed on the microscope stage.

The effective collecting area of the outer segments was calculated as previously described (Hestrin and Korenbrot, 1987). We assumed that the transverse specific density and the quantum efficiency of the cone outer segment are the same as that of the rod outer segment (Dartnall, 1972; Harosi, 1975). The average length and diameter at the base of the cone outer segments were 8.7 and $4.5 \mu \mathrm{m}$, respectively. Assuming that the peak absorbance of the salamander cones is at $620 \mathrm{~nm}$ (Attwell et al., 1982), we calculated that the average effective collecting area was $1.6 \mu \mathrm{m}^{2}$, at that wavelength. By comparing the response of cones to 620 $\mathrm{nm}$ and "white light," we found that the effective collecting area for "white light" was 15-fold larger than that of 620 light (see Cobbs and Pugh, 1987)

We did not measure the action spectra of the cones we recorded from.
Attwell et al. (1982) have found that the majority of tiger salamander cones have a peak absorbance at $620 \mathrm{~nm}$. Recently, Craig and Perry (1988) reported that, in addition to the red-sensitive cones, tiger salamander retina contain a smaller number of shortwave-sensitive cones. The photoresponse of these cones, however, is very different from that of red cones. On basis of the kinetics and the sensitivity to red light, we identified the photoreceptors we describe here as the red-sensitive cones.

\section{Results}

As has been previously reported, photocurrents measured in rods and cones differ in several respects: maximal amplitude, light sensitivity, and time course (reviewed by Cobbs and Pugh, 1986a; Baylor, 1987). We measured membrane current from single tiger salamander rods and cones using whole-cell recording. In darkness, the zero-current potential was about $-30 \mathrm{mV}$ for rods and about $-40 \mathrm{mV}$ for cones. The maximal amplitude of the photocurrent in cones was, on average, $25 \mathrm{pA}$ (range, 20$37, n=5$ ), whereas in rods it was $75 \mathrm{pA}$ (Hestrin and Korenbrot, 1987). The average light intensity that caused a suppression of $50 \%$ of the dark current in cones was 299 photons $/ \mu \mathrm{m}^{2}$ (range, $184-410, n=5$ ) and in rods it was 9 photons $/ \mu \mathrm{m}^{2}$ (range, 5.9$12.8, n=5$ ). The time to the peak of the response to dim light was, on average, $185 \mathrm{msec}$ (range, $175-210, n=5$ ) for the cones and $750 \mathrm{msec}$ for the rods (Hestrin and Korenbrot, 1987). These results are generally similar to those reported previously by others (Schnapf and McBurney, 1980; Cobbs et al., 1985; Nakatani and Yau, 1989).

\section{Kinetics of the photocurrent in response to intense flashes of light}

The purpose of the experiments reported here was to examine the initial response of cones to intense flashes and compare these responses to those of rods responses under similar light stimulation. Under tight-seal recording, the speed of the voltage clamp is limited by the series resistance and the capacitance of the cell's membrane. In the cones we studied, the membrane capacitance was in the range of 50-90 pF. This high value arises from the inner segment membrane and the membrane infolding of the outer segment which are continuous with the cell's plasma membrane. For the analysis of the response to bright light, we used data obtained from recordings that showed a series resistance (in the whole-cell mode) of about $10 \mathrm{M} \Omega$. In addition, some of the cells we recorded from were cones that were missing the nuclear region of the inner segment. These cones showed normal responses but had lower total capacitance. Further improvement in the speed of recording was sometimes obtained by employing series resistance compensation (50-70\%). The responses that are reported below were obtained from cells with a voltage-clamp time constant of about $1.0 \mathrm{msec}$. In rods, because cell capacitance is smaller, the voltage-clamp speed was typically 4-fold faster compared to that of cones.

The initial phase of the responses to a series of flashes of increasing intensity recorded from an isolated cone is shown in Figure 1 . The photocurrent elicited by the lowest intensity shown $\left(4.4 \times 10^{4}\right.$ photons $/ \mu \mathrm{m}^{2}$, bottom of Fig. 1) was time-delayed relative to the flash. The second half of the rising phase was well fit with a single-exponential function (dotted lines in Fig. 1). As the fraction of the bleached pigment increased, the time constant of this exponential decreased. The response to brighter flashes $\left(\geq 6 \times 10^{5}\right.$ photons $\left./ \mu \mathrm{m}^{2}\right)$ revealed an additional component that preceded the onset of the photocurrent. In response to the brightest flashes, the fast component was clearly biphasic. 

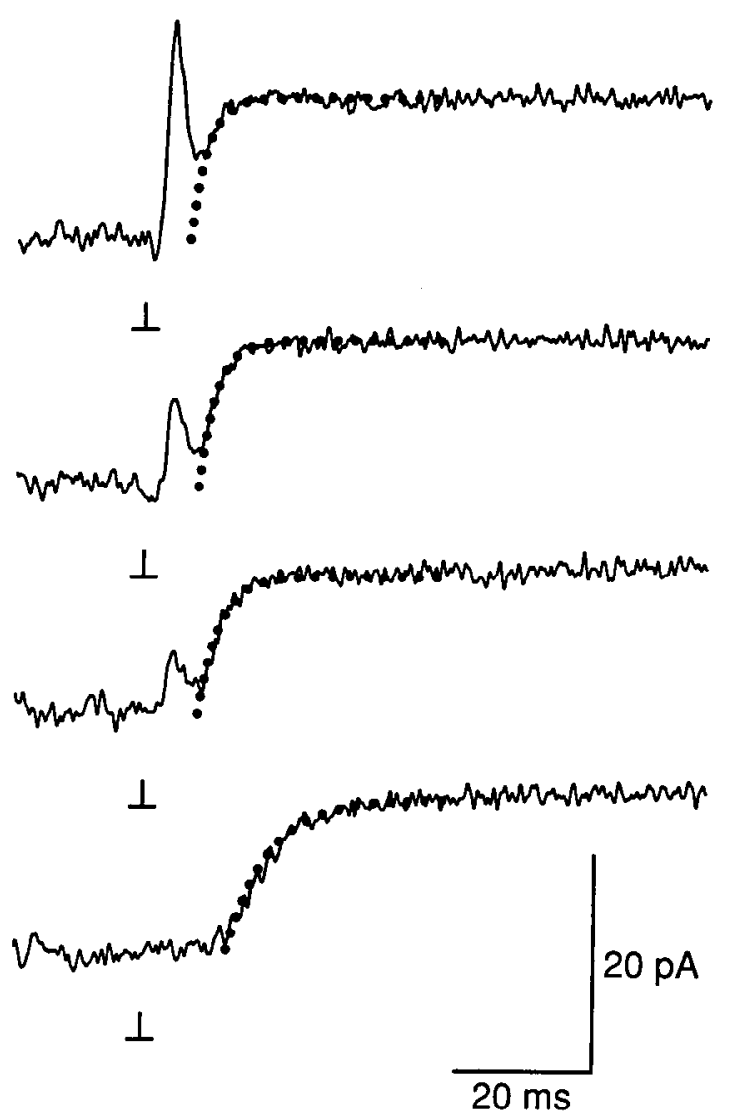

Figure 1. Response to intense flashes of light. The responses of a cone fragment (outer segment and elipsoid) to flashes of white light. The flash duration of $2.35 \mathrm{msec}$ was presented at the time marked underneath the traces. The light flashes delivered (from top to bottom): $2.9 \times 10^{6}$, $1.3 \times 10^{6}, 6.2 \times 10^{5}$, and $4.4 \times 10^{4}$ photons $/ \mu \mathrm{m}^{2}$. The response to the higher intensities has 2 separate kinetic components. A single-exponential function was fit to the fast component (dots superimposed on the current traces). The time constants of the fitted exponential functions were $2.27,2.46,3.12$, and $6.02 \mathrm{msec}$. The cell was held at $-40 \mathrm{mV}$; dark current, $15 \mathrm{pA}$. The membrane current was sampled at $20,000 \mathrm{~Hz}$ and filtered at $1000 \mathrm{~Hz}$.

It consisted of an inward current which rapidly developed into an outward one. The characteristics of this fast component, detailed below, suggest that it is a signal analogous to the ERP (early receptor potential) recorded extracellularly (Brown and Murakami, 1964; Cone and Pak, 1971; Hagins and Rupel, 1971) or intracellularly (Murakami and Pak, 1970; Hodgkin and O'Bryan, 1977). We termed this component the early receptor current (ERC). As shown in Figure 1, under very bright lights the time course of the photocurrent was obscured by the ERC. Thus, in order to analyze the saturation photocurrent in cones, it was necessary to show unambiguously that the ERC is not related to the photocurrent.

If the ERC, like the ERP, arises from charge displacement in photoexcited visual pigment, then this signal should exhibit 2 critical features: (1) The time course and amplitude of the ERC should be independent of the magnitude of the dark current, which reflects the conductance of the outer segment membrane; and (2) the amplitude of the ERC should be proportional to the number of photopigment molecules bleached by the stimulating light.

To test the independence between the ERC and the dark current, we stimulated a cone successively with 2 bright flashes
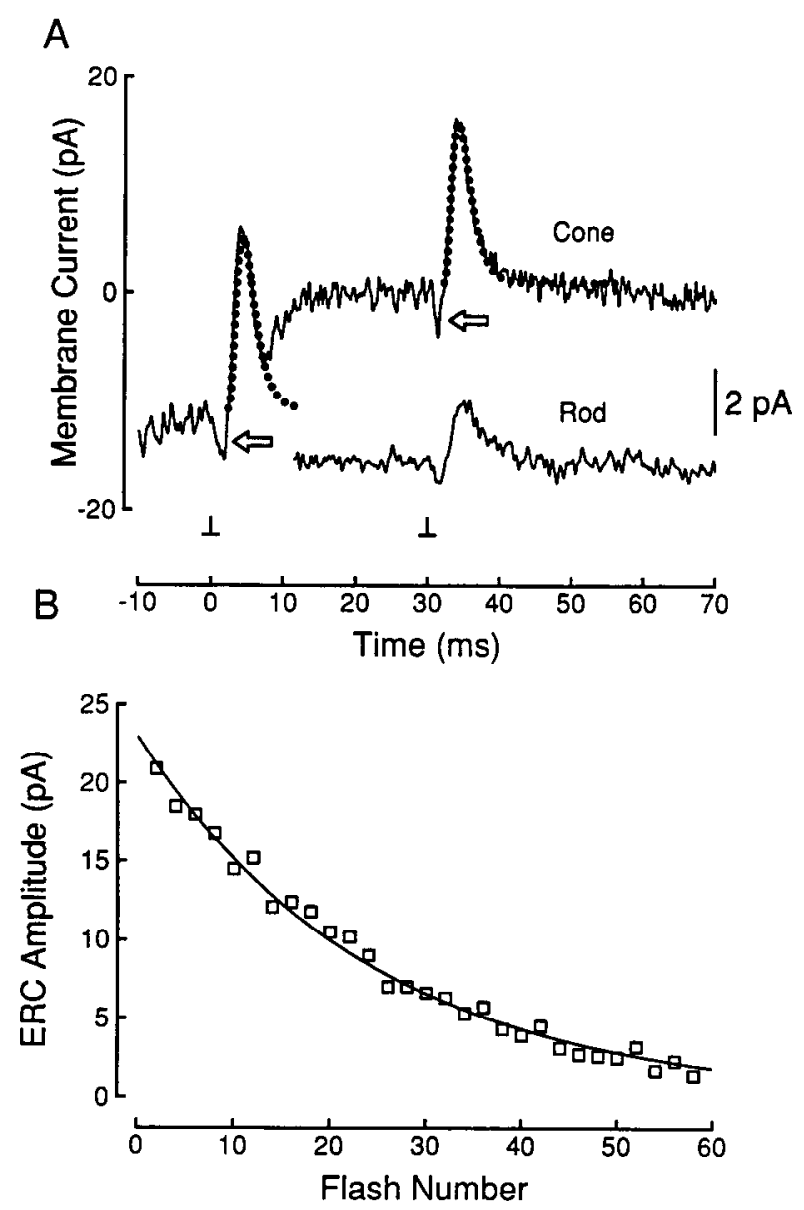

Figure 2. Properties of the early receptor current (ERC). A: Cone, Response of a cone to 2 consecutive stimuli. The flash photon density was $2.9 \times 10^{6}$ photons $/ \mu \mathrm{m}^{2}$. The response to the first flash consisted of both the ERC and the photocurrent. The response to the second flash consisted of the ERC alone. The ERC had 2 components: a dominant R2 (outward) and a smaller R1 (inward, marked by the arrows). The time course of the ERC evoked by the first flash was similar to that of the second ERC as indicated by the dots superpositioned on the data. Rod, An average of 10 responses of a light-adapted rod to bright flashes. The averaged response has similar time course to the cone ERC. $B$, The decay of the peak amplitude of the ERC. The data points are the peak amplitude of the ERC in response to a series of flashes. The intensity of each flash was $2.9 \times 10^{n}$ photons $/ \mu \mathrm{m}^{2}$. The continuous line is an exponential function fit to the data.

of the same intensity (Hodgkin and O'Bryan, 1977). In all cases tested, the first flash evoked an ERC followed by a saturating photocurrent (Fig. 2A). The second flash, presented during photocurrent saturation, also generated an ERC whose amplitude and time course were nearly identical to those elicited by the first flash. Thus, as expected, the current flowing through the light-sensitive channels in the outer segment had no effect on the ERC response.

Isolated rods should also exhibit ERC signals. However, because only a small fraction of the rod's photopigment resides in the plasma membrane (Ruppel and Hagins, 1973), the amplitude of the ERC of rods is expected to be smaller than that of cones. The ERC measured by averaging the response to 10 flashes presented to a rod is shown in Figure $2 \mathrm{~A}$. The rod was light-adapted, exhibiting no detcctablc dark current. As expected, the amplitude of the ERC in rods was small; however, 

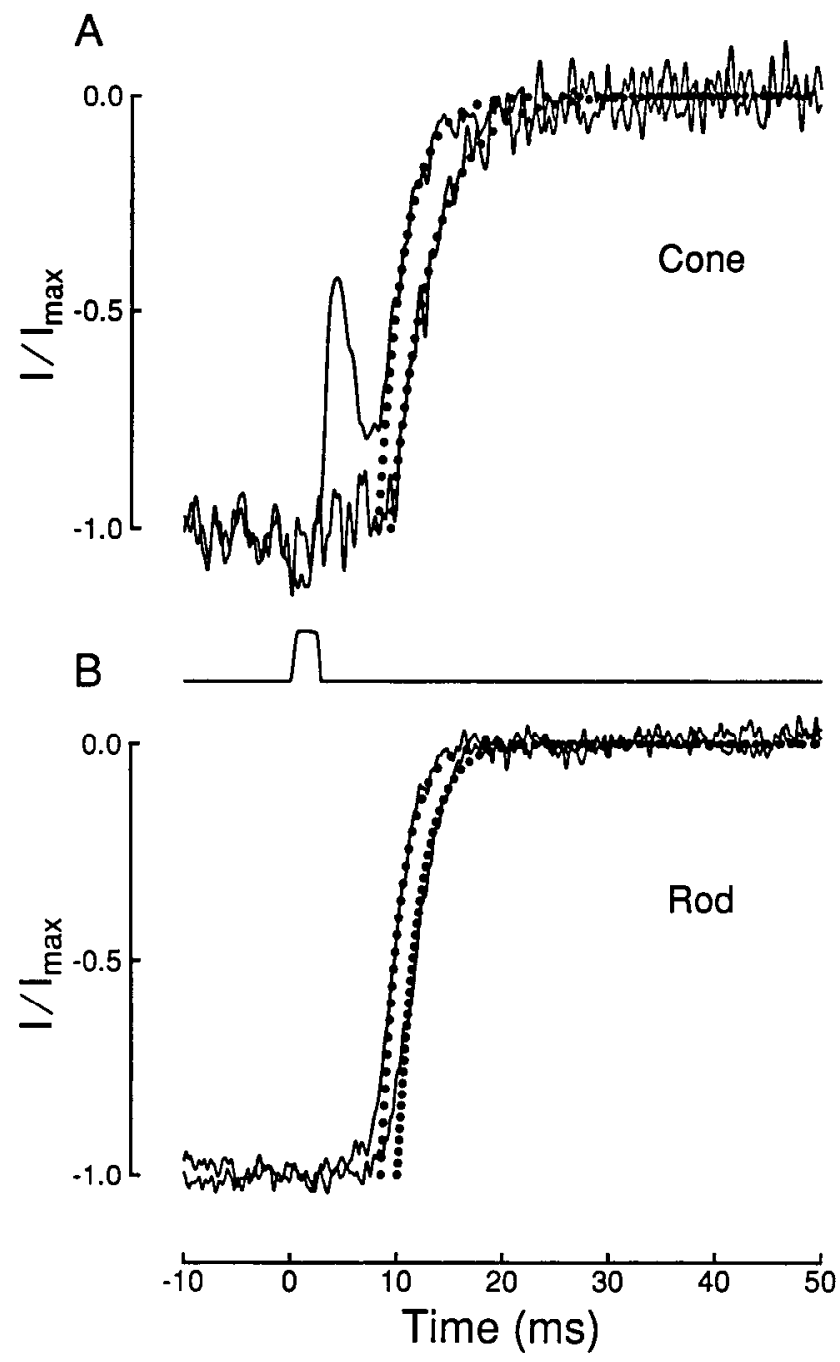

Figure 3. Comparison between the response of rod and cone at saturation and near-saturation levels. $A$, Normalized response of a cone to 2 intense flashes. Flash intensities were $1.3 \times 10^{6}$ and $2.1 \times$ $10^{5}$ photons $/ \mu \mathrm{m}^{2}$. The speed of the voltage clamp was $0.48 \mathrm{msec}$. The exponential fits (dotted line) had a time constant of $2.4 \mathrm{msec}$. The extrapolated delay of the brightest response was $8.6 \mathrm{msec}$ from the beginning of the flash (inset). $B$, Normalized response of an intact rod to 2 intense flashes. The flash intensities were $1.5 \times 10^{6}$ and $2.3 \times 10^{5}$ photons $/ \mu \mathrm{m}^{2}$. The exponential fits had a time constant of $1.9 \mathrm{msec}$. The extrapolated delay was $8.5 \mathrm{msec}$.

its time course was similar to that of the ERC measured in cones.

To measure the dependence of the ERC on the fraction of photopigment bleached, we compared the amplitude of the ERC elicited by a sequence of identical flashes. We found that the amplitude of the ERC decreased with each successive flash (Fig. $2 B$ ) and that after about 60 flashes, the ERC was almost undetectable. The relation between the ERC amplitude and flash number was well fit with a single-exponential function (solid line in Fig. 2B). This relation is expected if the amplitude of the ERC is proportional to the fraction of remaining unbleached photopigment (Hodgkin and O'Bryan, 1977). We used the exponential rate obtained from the fit to calculate that each flash bleached $4.2 \%$ of the pigment. This value was similar to the 2.6\% pigment bleached per flash predicted from the intensity

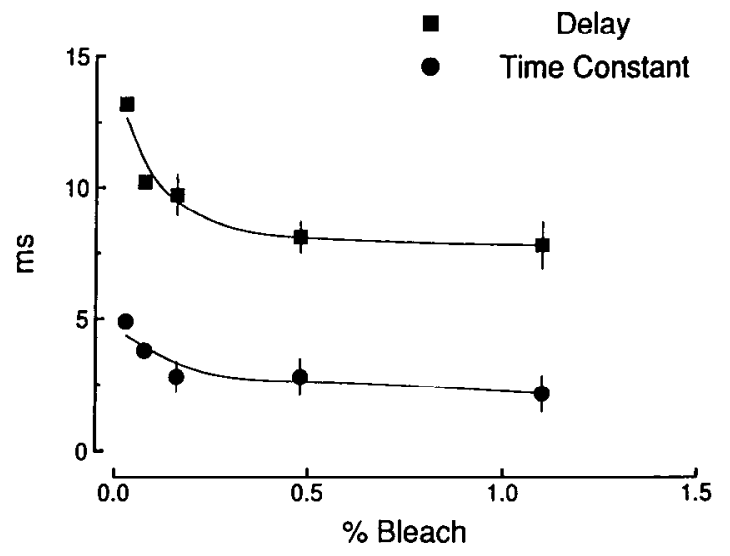

Figure 4. Limiting delay and velocity of the cone saturation photocurrent. The delay (squares) and velocity (circles) were measured as shown in Figure 3 from 3-7 single cones or cone fragments. Each cell was stimulated with light flashes of different intensities. The error bars indicate the standard deviation. The light intensity is given as the fraction of pigment bleached calculated as explained in Materials and Methods. The average speed of the clamp was $0.98 \mathrm{msec}$ as estimated from current responses to small voltage steps.

of the flash and the calculated cross section of the cone (see Materials and Methods).

\section{The saturation photocurrent}

Following the ERC, the cone photocurrent reached its maximum amplitude within about $10 \mathrm{msec}$ (Fig. $3 \mathrm{~A}$ ). The mathematical expression used to describe the dim flash response (Lamb et al., 1981) does not describe the response to bright-light stimulation. To characterize the kinetics of the saturating photocurrent, we fit an exponential function to the second half of the rising phase and extrapolated the fitted function to the baseline (Fig. 3A; Penn and Hagins, 1972; Cobbs and Pugh, 1987). We thus defined 2 parameters: a delay, defined as the time interval between the beginning of the flash and the intersection of the function with the baseline (Fig. $3 A$ ), and a velocity, defined as the rate of the exponential function.

We found that the delay and the velocity of the cone saturation photocurrent depended on the flash strength (Fig. 4). As the fraction of bleached pigment increased, the delay was shortened and the velocity increased. In response to light stimuli that bleached about $1-2 \%$ of the total cone pigment, the delay and the velocity reached limiting values. The limiting delay was 7.8 $+0.9 \mathrm{msec}$, and the limiting activation time constant was 2.1 $\pm 0.7 \mathrm{msec}$ (mean $\pm \mathrm{SD}, n=7$ ). The duration of the light "flashes" we used was $2.5 \mathrm{msec}$. The relatively long flash duration could distort the time course of the ERC, which showed little or no delay. However, the saturation response delay was about 3-fold longer than the flash duration and thus should show little distortion. Furthermore, the asymptotic behavior, under light saturation, of the response should be independent of the flash duration.

Cobbs and Pugh (1987) have previously measured the kinetics of the saturation photocurrent in tiger salamander rods. Their results show that in rods the limiting delay is $7 \mathrm{msec}$ and the limiting time constant is $2.8 \mathrm{msec}$. These results are virtually identical to those we measured from the cones. Indeed, Figure 3 illustrates the similarity of the early phase of the photocurrent in cones (Fig. 3A) and rods (Fig. 3B). It is remarkable that both 
receptor types display nearly identical kinetics when the fraction of bleached pigment is more than $0.1 \%$.

\section{Light-insensitive component}

Close examination of the time course of the saturation photocurrent in cones revealed a small, slow component in addition to the dominant fast component discussed above (Fig. 5). The time constant of the slow component was determined by fitting a double-exponential function to the second half of the rising phase of the bright-flash response. The time course of the slow component is shown in Figure 5. For a given cell, the same exponential function fit the responses generated by stimuli of different strengths. The amplitude and the time constant of the slow component were independent of light intensities above saturation. The time constant of the slow component was 95.4 $\pm 35 \mathrm{msec}$, and its amplitude (expressed as a percentage of dark current) was $6.1 \pm 2.3 \%$ (mean $\pm \mathrm{SD}, n=7$ ).

Previously, Cobbs and Pugh (1986b) have also reported on a slow component in the response of cones to saturating flashes of light. Recently, Nakatani and Yau (1989) characterized a Na$\mathrm{Ca}$ exchanger in salamander cone outer segments. The exchanger current identified by Nakatani and Yau (1989) was initiated by rapidly changing the solution bathing the outer segments of cones that were previously loaded with calcium. Under conditions of small calcium load, the time constant and amplitude of the $\mathrm{Na}-\mathrm{Ca}$ exchanger observed by Nakatani and Yau (1989) are similar to the time constant and amplitude we report here for the slow component. Furthermore, in rods the slow component following a saturating flash of light has been shown to exhibit a time constant and amplitude similar to those found by the solution-exchange method (Hodgkin et al., 1987, Fig. 3). Thus, it is likely that the slow component we observed in cones in response to saturating flashes of light is generated by the $\mathrm{Na}-$ $\mathrm{Ca}$ exchanger. Nonetheless, in order to make the unambiguous identification of the slow component as the $\mathrm{Na}-\mathrm{Ca}$ exchanger current, it will be important to show its dependence on external sodium.

\section{Discussion}

The current response of both rods and cones to bright flashes of light is characterized by 3 components. These are: the $E R C$, produced by charge displacement in visual pigment, a lightinsensitive component, that may reflect the activity of an electrogenic $\mathrm{Na}-\mathrm{Ca}$ exchanger and the saturation photocurrent, caused by the closure of the light-sensitive channels.

\section{Early receptor current ( $E R C$ )}

The ERC is the current analog of the ERP recorded previously from rods and cones (Brown and Murakami, 1964; Cone and Pak, 1971; Rupel and Hagins, 1973; Hodgkin and O'Bryan, 1977). We found that the cone's ERC is composed of a small inward current followed by a large outward current, these components are termed $R 1$ and $R 2$, respectively. The ERP recorded previously from rod and cone photoreceptors is composed of 2 similar components. In rods, the time course and temperature dependence of the R2 component suggest that this component is related to the formation of MetaII (Cone and Cobbs, 1969), thought to be the intermediate state of excited rhodopsin ( $\left.\mathrm{Rh}^{*}\right)$ which activates the G-binding protein (Stryer, 1986). In cones, the kinetics of formation of opsin photointermediates has not been measured by spectrophotometry. However, the similarity

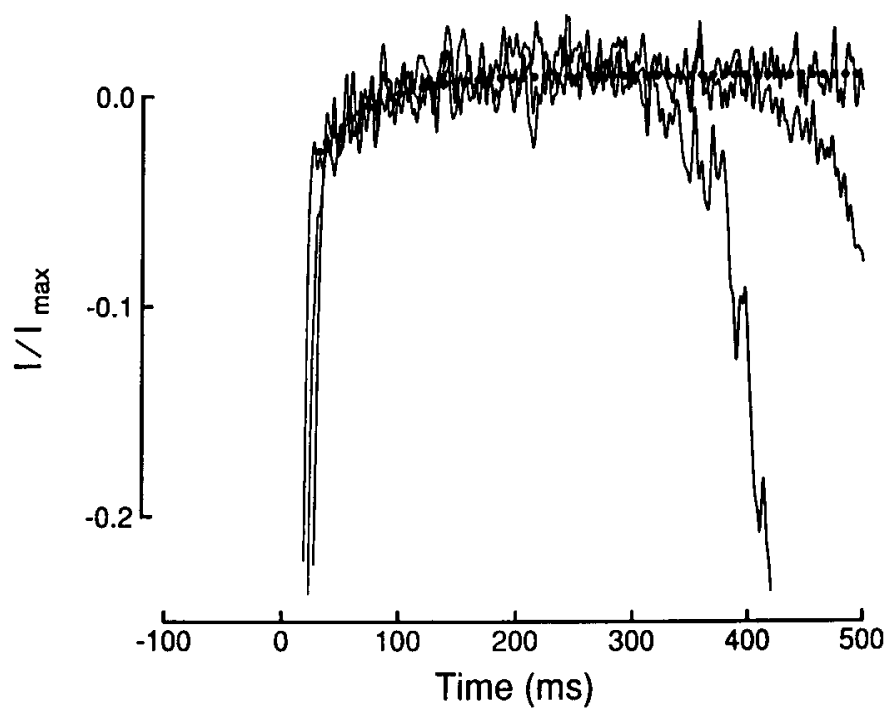

Figure 5. The light-insensitive component. The normalized slow component of responses to bright flashes of light. Three responses of the same cone to flashes of $4.03 \times 10^{4}, 8.82 \times 10^{4}$, and $2.64 \times 10^{5}$ photons/ $\mu \mathrm{m}^{2}$. The dotted line traces a single-exponential function with a time constant of $73.5 \mathrm{msec}$ and whose initial magnitude was $3.9 \%$ of the dark current.

of the $\mathrm{R} 2$ kinetics in cones and rods suggests that the kinetics of the formation of the cone's $\mathrm{Rh}^{*}$ is similar to that of rods.

Wc calculate that the charge displaced across the membrane field per bleached cone photopigment is about 0.08 electronic charges. The electronic charge displaced per bleached photopigment has been previously estimated to be 0.2 electronic charges in toad rods (Hochstrate et al., 1982) and 0.12 electronic charges in turtle red-sensitive cones (Hodgkin and O'Bryan, 1977). It is thus apparent that the kinetics and magnitude of the charge displacement are generally conserved between species and receptor types. These findings probably reflect the fact that opsins from rods and cones of various species are highly homologous, as revealed by sequencing of the opsin genes (Applebury and Hargrave, 1986; Nathans, 1987).

\section{Saturation photocurrent}

The cone saturation photocurrent is characterized by a delay between the flash and the onset of the photocurrent and by the exponential time constant with which the photocurrent approaches its maximum value. At saturation, the limiting delay was $7.8 \mathrm{msec}$ and the limiting time constant was $2.1 \mathrm{msec}$. Remarkably, these parameters are virtually identical (within experimental errors) to those found in rods of the same species (Cobbs and Pugh, 1987). Furthermore, the fraction of pigment bleaching that was required to produce the rate-limiting kinetics was comparable in rods and cones.

Cobbs and Pugh (1987) suggested that in the rods the limiting delay in the saturation photocurrent results from multiple cooperative kinetic steps between rhodopsin activation and the activation of the PDE, whereas its limiting time constant reflects the maximum rate of cGMP hydrolysis or the closing rate of the light-sensitive channels. Their analysis is generally compatible with known physiological and biochemical properties of rod photoreceptors. In salamander rods, the lifetime of the lightsensitive channel appears to be about $2 \mathrm{msec}$ (Gray and Attwell, 1985; Karpen et al., 1988), and similar observations have been 
made on the lifetimes of the light-sensitive channel in rods from other species (Bodoia and Detwiler, 1985; Fesenko et al., 1985; Haynes et al., 1986; Matthews, 1986). Thus, in the rod, the limiting time constant could be due to the closure kinetics of the light-sensitive channels (Cobbs and Pugh, 1987). These data, however, do not preclude the possibility that the limiting time constant reflects the maximal speed of reduction in the concentration of cytoplasmic cGMP (Cobbs and Pugh, 1987).

Haynes and Yau (1987) have reported that the cGMP-activated channels found in catfish cone outer segment have a burst duration of about $3 \mathrm{msec}$. The lifetime of the light-sensitive channels in cones has not been reported. Preliminary noise analysis of the light-sensitive current in salamander cones revealed a high-frequency Lorentzian component that is similar to that observed in rods of the same species, suggesting that the lifetime of the cone's light-sensitive channel is about $2 \mathrm{msec}$ (S. Hestrin and J. I. Korenbrot, unpublished observations). Although the lifetime of the light-sensitive channels in rods and cones might be similar, the current-voltage relationships of these channels are different. Haynes and Yau (1985) have shown that as the membrane potential is hyperpolarized, the current through the cGMP-activated channels in cones is increased. In contrast, the current-voltage relation $(I-V)$ of the light-sensitive conductance of rods is nearly flat in the physiological range of membrane potential (Baylor and Nunn, 1986). The importance of the difference between the $I-V$ of rods and cones is unclear.

Under light saturation the interaction between the cone $\mathrm{Rh}^{*}$ and cone G-binding protein would not be rate limiting, and thus, as in rods (Cobbs and Pugh, 1987), the delay of about 8 msec is likely to be related to multiple cooperative kinetic steps linking the G-binding protein to the activation of the PDE. Without further details on the biochemical rates of the cGMP cascade enzymes in cones, it is not possible to assign the limiting delay and the limiting time constant to specific reactions. Nonetheless, our results suggest that the excitation of rods and cones has in common at least 2 kinetic steps that are quantitatively similar.

Photoreceptor excitation is a multistep process, and therefore our experiments, done under intense light, do not entirely rule out the importance of the excitation process in generating the rod-cone differences of the dim-flash response. For example, it is possible that under dim-flash stimulation, the interaction of $\mathrm{Rh}^{*}$ with the $\mathrm{G}$-binding protein is a rate-limiting step, generating some of the rod-cone difference.

Nakatani and Yau (1989) suggested that the difference between rod and cone light responses is generated by shortened lifetime of active intermediate in the cone transduction cascade. A similar hypothesis which is compatible with our observations would be that the principal proteins which participate in the excitation-the photopigments, the G-binding proteins, the PDEs, and the cGMP-sensitive-channels are similar in salamander rods and cones in both properties and number per unit volume. The differences between rods and cones would then be generated from differences in the deactivation processes (Nakatani and Yau, 1989). Specifically, the differences might be related to the turn-off of the PDF or the regeneration of cytoplasmic cGMP. Thus, this hypothesis predicts that the guanylate cyclase of cones operates with a higher turnover rate than that of rods, or that in cones the PDE turns off in a faster rate than the PDE in rods.

\section{References}

Applebury ML, Hargrave PA (1986) Molecular biophysics of the visual pigments. Vision Res. 26:1881-1895.

Attwell D, Werblin FS, Wilson M (1982) The properties of single cones isolated from the tiger salamander retina. J Physiol (Lond) 328: 259-283.

Baylor DA (1987) Photoreceptors signals and vision. Invest Ophthalmol Sci 28:34-49.

Baylor DA, Nunn BJ (1986) Electrical properties of the light-sensitive conductance of rods of the salamander Ambystoma tigrinum. I Physiol (Lond) 371:115-145.

Bodoia RD, Detwiler PB (1985) Patch-clamp recording of the lightsensitive dark noise in retinal rods from the lizard and frog. J Physiol (Lond) 376:183-216.

Booth DP, Hurwitz RL, Lolley RN (1986) Characterization of cyclic nucleotide phosphodiesterase of lizard cone photoreceptors. Invest Ophthalmol Vis Sci (Suppl) 27:217.

Brown KT, Murakami M (1964) A new receptor potential of the monkey retina with no detectable delay. Nature 201:626-628.

Cobbs WH, Pugh EN (1986a) Visual transduction in vertebrate rods and cones: a tale of two transmitters, calcium and cyclic GMP. Vision Res 26:1613-1643.

Cobbs WH, Pugh EN (1986b) Two components of outer segment membrane current in salamander rods and cones. Biophys J 49:280a.

Cobbs WH, Pugh EN (1987) Kinetics and components of the flash photocurrent of isolated retinal rods of the larval salamander, $\mathrm{Am}$ bystoma tigrinum. J Physiol (Lond) 394:529-572.

Cobbs WH, Barkdoll AE III, Pugh EN (1985) Cyclic GMP increases photocurrent and light sensitivity of retinal cones. Nature 317:64-66.

Cone RA, Cobbs WH (1969) Rhodopsin cycle in the living eye of the rat. Nature 221:820-822.

Cone RA, Pak WL (1971) The early receptor potential. In: Handbook of sensory physiology, Vol 1 (Loewnstein WL, ed), pp 345-365. Berlin: Springer-Verlag.

Craig AJ, Perry RJ (1988) Difference in absolute sensitivity and response kinetics between red-, blue- and ultraviolet-sensitive isolated salamander cones. J Physiol (Lond) 407:83p.

Dartnall HJA (1972) Photosensitivity. In: Photochemistry of vision (Dartnall HJA, ed), pp 122-145. New York: Springer-Verlag.

Fesenko EE, Stanislav SK, Lyubarsky AL (1985) Induction by cyclic GMP of cationic conductance in plasma membrane of retinal rod outer segment. Nature 313:310-313.

Gillespie PG, Beavo JA (1988) Characterization of a bovine cone photoreceptor phosphodiesterase purified by cyclic GMP-sepharose chromatography. J Biol Chem 263:8133-8141.

Gray P, Attwell D (1985) Kinetics of light-sensitive channels in vertebrate photoreceptors. Proc R Soc Lond [Biol] 223:379-388.

Grunwald GB, Gierschik M, Nirenberg A, Spiegel A (1986) Detection of alpha-transducin in retinal rods but not cones. Science 231:856859.

Hagins WA, Ruppel H (1971) Fast photoelectric effects and the properties of vertebrate photoreceptors as electric cables. Fed Proc 30 856-859.

Harosi FI (1975) Absorption spectra and linear dichroism of some amphibian photoreceptors. J Gen Physiol 66:357-382.

Haynes LW, Yau KW (1985) Cyclic GMP-sensitive conductance in outer segment membranes of catfish cones. Nature 317:61-64.

Haynes LW, Yau KW (1987) Single cGMP-activated channcl activity recorded from excised cone membrane patches. Biophys J 51:18a.

Haynes LW, Kay AR, Yau KW (1986) Single cGMP-activated channel activity in excised patches of rod outer segment membranes. Nature 321:66-70.

Hestrin S, Korenbrot JI (1987) Effects of cyclic GMP on the kinetics of the photocurrent in rods and in detached rod outer segments. $J$ Gen Physiol 90:527-551.

Hochstrate P, Lindau M, Ruppel H (1982) On the origin and the signal-shaping mechanism of the fast photosignal in the vertebrate retina. Biophys J 38:53-61.

Hodgkin AL, O'Bryan PM (1977) Internal recording of the early receptor potential in turtle cones. J Physiol (Lond) 267:737-766.

Hodgkin AL, McNaughton PA, Nunn BJ (1987) Measurement of 
sodium-calcium exchange in salamander rods. J Physiol (Lond) 391: 347-370.

Hurwitz RL, Bunt-Milam AH, Change ML, Beavo JA (1985) cGMP phosphodiesterase in rod and cone outer segments of the retina. J Biol Chem 260:568-573.

Karpen JW, Zimmerman AL, Stryer L, Baylor DA (1988) Gating kinetics of the cyclic-GMP-activated channel of retinal rods: flash photolysis and voltage-jump studies. Proc Natl Acad Sci USA 85: 1287-1291.

Korenbrot JI, Miller DL (1986) Calcium ions act as modulators of intracellular information flow in retinal rod photoreceptors. Neurosci Res 4:S11-S34.

Lamb TD, MacNaughton P, Yau KW (1981) Spatial spread of activation and background desensitization in toad rod outer segments. $J$ Physiol (Lond) 319:463.

Lerea CL, Somer DE, Hurley JB, Klock IB, Bunt-Milam AH (1986) Identification of specific transducin $\alpha$ subunits in retinal rod and cone photoreceptors. Science 234:77-80.

Matthews G (1986) Comparison of the light-sensitive and the cyclic GMP-sensitive conductances of the rod photoreceptor: noise characteristics. J Neurosci 6:2521-2526.

Murakami M, Pak WL (1970) Intracellularly recorded early receptor potential of the vertebrate photoreceptors. Vision Res 10:965-975.

Nakatani K, Yau KW (1989) Sodium-dependent calcium extrusion and sensitivity regulation in retinal cones of the salamander. J Physiol (Lond) 409:525-548.
Nathans J (1987) Molecular biology of visual pigments. Annu Rev Neurosci 10:163-194.

Orlov NY, Kalinin EV, Orlova TG, Freidin AA (1988) Properties and content of cyclic nucleotide phosphodiesterase in photoreceptor outer segments of ground squirrel retina. Biochem Biophys Acta 954: 325-335.

Penn RD, Hagins WA (1972) Kinetics of the photocurrent of retinal rods. Biophys J 12:1073-1094.

Pugh EN (1987) The nature and identity of the internal excitational transmitter of vertebrate phototransduction. Annu Rev Physiol 49: 715-741.

Rodieck RW (1973) The vertebrate retina. San Francisco: WH Freeman.

Ruppel H, Hagins WA (1973) Spatial origin of the fast photovoltage in retinal rods. In: Biochemistry and physiology of visual pigments. (Langer H, ed), pp 257-261. Berlin: Springer-Verlag.

Schnapf J, McBurney R (1980) Light-induced changes in membrane current in cone outer segments of tiger salamander and turtle. Nature 287:239-241.

Stryer L (1986) Cyclic GMP cascade of vision. Annu Rev Neurosci 9:87-119.

Torre V, Matthews HR, Lamb, TD (1986) Role of calcium in regulating the cyclic GMP cascade of phototransduction in retinal rods. Proc Natl Acad Sci USA 83:7109-7113. 